\title{
The cellular role of Drosophila hyperplastic discs gene
}

\author{
Y.A. Galimova ${ }^{1}$, E.U. Bolobolova ${ }^{2}$, A.S. Khruscheva ${ }^{2}$, N.V. Dorogova ${ }^{2 *}$ \\ ${ }^{1}$ Institute of Molecular and Cellular Biology SB RAS, Novosibirsk, Russia, Novosibirsk, Russia \\ ${ }^{2}$ Institute of Cytology and Genetics SB RAS, Novosibirsk, Russia \\ *e-mail:dorogova@bionet.nsc.ru
}

Key words: tumor suppressor Hyd, ubiquitin ligase, Drosophila

Motivation and Aim: The tumor suppressor Hyd (hyperplastic discs), also known as EDD (E33 isolated by differential display) or Rat100, is a member of the ubiquitin ligase (E3) family containing the HECT domain (homologous to E6-associated protein carboxyl terminus) that provides specific ubiquitin-mediated proteolysis. A highly conservative ubiquitin-dependent proteolysis system controls the degradation of many key regulatory proteins. The tumor suppressor nature of Hyd protein was supported by the studies in mammalians [1]. Hyd is required for the regulation of cell proliferation during development [2], and mutations in the hyd gene resulting in developmental abnormalities that include adult sterility caused by germ cell defects [3].

Methods and Algorithms: We used Bloomington stocks: kniri-1 hyd $d^{15} e^{1 / T M 3, S b^{1}}$ (3718) and $y^{1} w^{1118}$; PBac $\{3 H P y+\} h y d^{C 017} / T M 3, S^{1} \operatorname{Ser}^{l}$ (16256) as the source of hyd alleles. Isolated testes and ovaries were examined by the methods of light, fluorescence and electron microscopy.

Results: The role hyd in Drosophila germinal line has been studied. It is shown that mutations of this gene cause multiple cell division abnormalities in spermatogenesis, which included: abnormal chromosome segregation and absence of meiotic cytokinesis resulting in formation of polyploid spermatids, and also defects in spermatid elongation. However, these abnormalities do not lead to hyperplasia of generative tissue. In contrast, in oogenesis, this gene manifests the function of a tumor growth suppressor, the disturbances of which cause an excessive number of cell divisions and overproliferation. Conclusion: The question arises, why the same protein has different effects on the cell division in the generative tissue of males and females. Even earlier studies have shown that tumor suppressor genes can function in many tissues, but only in some of them they cause hyperplasia [4]. Probably, in oogenesis, the hyd gene manifests as a tumor suppressor, and in spermatogenesis as a ubiquitin ligase.

\section{References}

1. Cho E. et al. (2006). Delineation of a Fat tumor suppressor pathway. Nat. Genet. 38:1142-1150.

2. Mansfield, E.A. et al. (1994). Genetic and molecular analysis of hyperplastic discs, a gene whose product is required for regulation of cell proliferation in Drosophila melanogaster imaginal discs and germ cells. Dev. Biol. 165(2):507-526.

3. Callaghan M.J. et al. (1998). Identification of a human HECT family protein with homology to the Drosophila tumor suppressor gene hyperplastic discs. Oncogene. 17:3479-3491.

4. Gateff E. (1994). Tumor suppressor and overgrowth suppressor genes of Drosophila melanogaster: Developmental aspects. Int. J. Dev. Biol. 38:565-590. 